\title{
Can an ensemble transform Kalman filter predict the reduction in forecast-error variance produced by targeted observations?
}

\author{
By S. J. MAJUMDAR ${ }^{1 *}$, C. H. BISHOP' ${ }^{1}$, B. J. ETHERTON ${ }^{1}$, I. SZUNYOGH ${ }^{2}$ and Z. TOTH ${ }^{2}$ \\ ${ }^{\mathrm{I}}$ The Pennsylvania State University, USA \\ ${ }^{2}$ National Centers for Environmental Prediction, USA
}

(Received 2 January 2001; revised 1 June 2001)

\begin{abstract}
SUMMARY
The ensemble transform Kalman filter (ET KF) is currently used at the National Centers for Environmental Prediction (NCEP) to identify deployments of aircraft-borne dropwindsondes that are likely to significantly improve 1-3 day forecasts of winter storms over the continental United States. It is unique among existing targeted observing strategies in that it attempts to predict the reduction in forecast-error variance associated with each deployment of targeted observations. To achieve this, the ET KF predicts the variance of 'signals' for each feasible deployment, where a signal represents the difference between two forecasts, initialized with and without the targeted observations. For linear forecast-error evolution, the signal variance is equal to the reduction in forecasterror variance, provided that observation- and background-error covariances are accurately specified and identical to those produced by the operational data-assimilation scheme. However, background-error covariances assumed by the ET KF are both imperfect and different from the imperfect error covariances used in NCEP's 3D-Var dataassimilation scheme, and hence their signal statistics are likely to differ.

In spite of these differences, a linear relationship of positive gradient is found to exist between the ET KF signal variance and the sample variance of NCEP signal realizations at both the targeted analysis and forecast verification times, for 30 forecasts from the 2000 Winter Storm Reconnaissance Program. This relationship enables the NCEP signal variance to be predicted by the ET KF, via a statistical rescaling that corrects the ET KF's current over-prediction of signal variance magnitude. A monotonically increasing relationship is also found to exist between the NCEP signal variance and the reduction in NCEP forecast-error variance. The ET KF signal variance predictions can be used to make quantitative estimates of the forecast-error-variance reducing effect of targeted observations. Potential benefits include (i) making rapid decisions on when and where to deploy targeted observations, (ii) warning operational data quality-control schemes against the rejection of observational data if the signal variance is large, and (iii) estimating the likelihood of economic benefit due to any future deployment of observations.
\end{abstract}

KEYwORDS: Data assimilation ET KF Forecast-error covariance Signal covariance

\section{INTRODUCTION}

The ensemble transform Kalman filter (ET KF) technique for adaptive sampling or 'targeting' has been used at the National Centers for Environmental Prediction (NCEP) during the quasi-operational 1999 and 2000 Winter Storm Reconnaissance (WSR) Programs (Toth et al. 1999; Szunyogh et al. 2000), and the operational 2001 WSR program (Toth et al. 2001). The main aim of these programs has been to improve 1-3 day forecasts of winter storms over the continental United States. Aircraft have been deployed to release Global Positioning System dropwindsondes in atmospheric regions over the northern Pacific Ocean; these data were then transmitted to NCEP for use in the next operational analysis-forecast cycle. Using ensemble forecasts issued 3-6 days prior to the verification time, the ET KF has been used to estimate the reduction in forecast-error variance $\dagger$ within a pre-selected circular verification region of radius $1000 \mathrm{~km}$, for all feasible aircraft flight tracks. The track that maximized this error variance reduction was deemed optimal. The ET KF theory is explained in Bishop et al. (2001), and the ET KF products used during adaptive observing programs are described

* Corresponding author: RSMAS/MPO, University of Miami, 4600 Rickenbacker Causeway, Miami FL 331491098, USA. e-mail: smajumdar@rsmas.miami.edu

+ Throughout this paper, the variance of a particular quantity is defined as the mean square of independent realizations of that quantity.

(C) Royal Meteorological Society, 2001. 
in Majumdar et al. (2001) (hereafter MEA). This paper focuses on the ability of the ET KF to provide quantitative estimates of the reduction of operational analysis and forecast-error variance for any deployment of dropwindsondes.

If the ET KF is a successful predictor in this regard, one would have confidence in its ability to identify optimal times and locations for the deployment of targeted observations. Such a technique would also potentially be useful in the near future to deploy proposed new instruments such as aerosondes (Holland et al. 1992) and other unmanned aerial vehicles to augment the existing routine observational network. Here, we refer to the routine observational network as the set of non-adaptive observations that cannot be varied at will, such as those taken by satellites, rawinsonde balloons and regular aircraft. Furthermore, the ET KF could in theory be used to identify regions where data quality-control schemes should not necessarily reject new data even if they differ considerably from the first-guess field. Estimates of forecast-errorvariance reduction may also be coupled with a simple economic model to estimate the potential financial benefit of forecast improvement within a populated area, due to any deployment of targeted observations.

A signal realization is defined as the difference between an analysis (or forecast) that assimilates both routine and targeted observations, and an analysis (or forecast) that uses only the routine observations. The ET KF estimates the variance of these signals, for a theoretically infinite number of realizations produced by an infinite number of independent background and observation errors. If the error covariances assumed by the ET KF and the operational data-assimilation scheme were both accurate and identical, and the errors propagated linearly, then this signal variance would be mathematically equal to the reduction in forecast-error variance imparted by the targeted observations (see proof in Bishop et al. (2001)). However, errors evolve nonlinearly, and signals produced by an ET KF data-assimilation scheme and by NCEP's 3D-Var data-assimilation scheme (Parrish and Derber 1992) may not resemble each other for several reasons. Firstly, the ET KF's ensemble-based background-error statistics and the quasi-isotropic background-error statistics assumed by 3D-Var can be very different and, moreover, both sets of error statistics may be inaccurate. Secondly, the operational implementation of the ET KF targeting strategy (MEA) only crudely accounts for observations to be taken at times between the ensemble initialization time (shortly before the flight decision is made) and the future targeted observing time. It does this by linearly transforming the ensemble perturbations to produce new perturbations that are orthonormal with respect to an inverse analysis uncertainty norm and consistent with global estimates of analysis-error variance (details are given in MEA). Thirdly, the model trajectory used in the ET KF (based on older European Centre for Medium-Range Weather Forecasts (ECMWF) and NCEP forecasts) may differ from the NCEP forecast trajectory initialized at the time that the targeted observations are taken. The ability of the ET KF and its predecessor (ensemble transform technique, see Bishop and Toth (1999)) to select successful target regions in adaptive sampling programs, despite these limitations, is documented in Szunyogh et al. (1999a,b, 2000) and Toth et al. (1999, $2000,2001)$.

Szunyogh et al. (1999a) demonstrated that the evolution of NCEP signal realizations and forecast improvements were both strongly related to the amplification rate of ensemble perturbations during FASTEX ${ }^{*}$. In this paper, we build on the promise of Szunyogh $e t$ al.'s results by testing the ability of the ET KF signal variance to make quantitative predictions of the variance of evolving NCEP signals and the reduction of

${ }^{*}$ Fronts and Atlantic Storm-Track EXperiment. 
error variance in NCEP's forecasts, due to the targeted observations. While it can be deduced (e.g. from Eq. (17b) of Bishop et al. 2001) that the ET KF signal variance amplification is closely related to the ensemble amplification rate, we suggest that the ET KF signal variance provides a more theoretically rigorous means of making quantitative predictions. To summarize, in this paper we seek the answers to two questions, using data from the WSR00 field program:

(i) Does a linear, increasing relationship exist between the ET KF signal variance and the variance of operational NCEP signal realizations? If so, the ET KF can be used to estimate the NCEP signal variance via a statistical rescaling factor if their magnitudes differ.

(ii) Does a similar relationship exist between the signal variance of NCEP forecasts and the reduction in NCEP forecast-error variance? If so, the two relationships can be combined to give quantitative ET KF estimates of the forecast-error reducing effect of targeted observations within verification regions of interest.

In section 2 of this paper, we illustrate the similarities and differences between evolving signals produced by the ET KF and NCEP 3D-Var data-assimilation schemes. In section 3, we answer question (i) by grouping together NCEP signal realizations to calculate their sample variance. Tests are performed to determine the relationship between the NCEP sample signal variance and the predicted ET KF signal variance at the analysis and verification times. In section 4, question (ii) is tested at the verification time, to determine whether a relationship between the ET KF signal variance and NCEP reduction in forecast-error variance can be deduced. Concluding remarks are given in section 5 .

\section{ET KF AND 3D-VAR SIGNAL COVARIANCES}

\section{(a) Differences between $E T K F$ and $3 D$-Var signals}

The implementation of the ET KF targeting strategy during NCEP's adaptive observing programs is described in MEA. To summarize, the ET KF uses ensemble perturbations initialized at time $t_{\mathrm{i}}$ to estimate the covariance of 'signals' imparted by targeted observations at a future analysis or targeting time $t_{\mathrm{a}}$, which is typically 24-60 hours after $t_{\mathrm{i}}$ (Fig. 1). A signal vector $\mathrm{s}^{q}(t)$ valid at time $t \geqslant t_{\mathrm{a}}$ is defined as the difference between an analysis (or forecast) vector $\mathrm{x}^{q}(t)$ that assimilates the routine observations plus the $q$ th set of targeted data contained in the vector $\mathrm{y}^{q}$ at time $t_{\mathrm{a}}$, and a routine analysis (or forecast $) \mathbf{x}^{\mathbf{r}}(t)$ that is identical in every respect except that all targeted data are ignored. It is expressed mathematically as

$$
\begin{aligned}
\mathbf{s}^{q}(t) & =\mathbf{x}^{q}(t)-\mathbf{x}^{\mathrm{r}}(t) \\
& =\mathbf{M} \mathbf{P}^{\mathrm{r}}\left(t_{\mathrm{a}}\right) \widehat{\mathbf{H}}_{\mathbf{a}}^{q \mathrm{~T}}\left(\widehat{\mathbf{H}}_{\mathbf{a}}^{q} \mathbf{P}^{\mathrm{r}}\left(t_{\mathrm{a}}\right) \widehat{\mathbf{H}}_{\mathrm{a}}^{q \mathrm{~T}}+\widehat{\mathbf{R}}^{q}\right)^{-1}\left(\mathbf{y}^{q}-\widehat{\mathbf{H}}_{\mathrm{a}}^{q} \mathbf{x}^{\mathrm{r}}\left(t_{\mathrm{a}}\right)\right),
\end{aligned}
$$

where $\mathbf{P}^{\mathrm{r}}\left(t_{\mathrm{a}}\right)$ is the routine-analysis error covariance matrix, and $\widehat{\mathbf{H}}_{\mathrm{a}}^{q}$ is the observation operator corresponding to the targeted observational network; $\widehat{\mathbf{R}}^{q}$ is the observationalerror covariance matrix associated with targeted data, and $\mathbf{M}$ is the linearized operator that propagates the signal from analysis time $t_{\mathrm{a}}$ to future time $t$. The structure of the signal depends on the difference between the targeted observations and the routineanalysis estimate interpolated onto the targeted observation sites (known as the innovation vector), and the observation-error and routine-analysis error covariances assumed by the data-assimilation scheme. Both the analysis and targeted observation errors may 


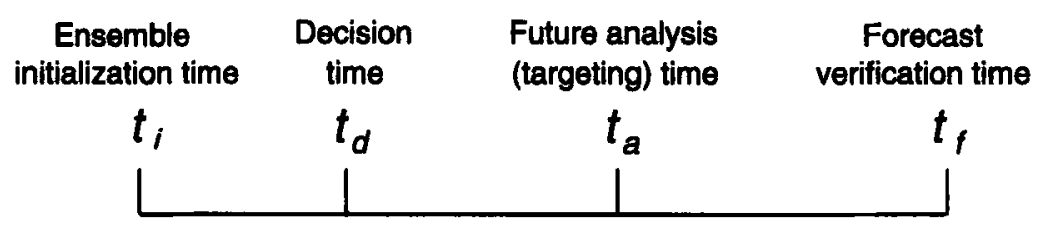

Figure 1. Times involved in the decision making process. Based on an ensemble initialized at time(s) $t_{i}$, a decision is made at time $t_{\mathrm{d}}$ to deploy adaptive observational resources at the future analysis time $t_{\mathrm{a}}$, to improve a forecast (initiated at $t_{\mathrm{a}}$ ) valid within a verification region at time $t_{\mathrm{f}}$.

be viewed as resulting from independent stochastic processes. Similarly, signal realizations can be viewed as stochastic processes whose variations are described by a signal covariance matrix, which is expressed as

$$
\begin{aligned}
\mathbf{S}^{q}(t) & =\left\langle\left(\mathbf{x}^{q}(t)-\mathbf{x}^{\mathrm{r}}(t)\right)\left(\mathbf{x}^{q}(t)-\mathbf{x}^{\mathrm{r}}(t)\right)^{\mathrm{T}}\right\rangle \\
& =\mathbf{M P}^{\mathrm{r}}\left(t_{\mathrm{a}}\right) \widehat{\mathbf{H}}_{\mathrm{a}}^{q \mathrm{~T}}\left(\widehat{\mathbf{H}}_{\mathrm{a}}^{q} \mathbf{P}^{\mathrm{r}}\left(t_{\mathrm{a}}\right) \widehat{\mathbf{H}}_{\mathrm{a}}^{q \mathrm{~T}}+\widehat{\mathbf{R}}^{q}\right)^{-1} \widehat{\mathbf{H}}_{\mathrm{a}}^{q} \mathbf{P}^{\mathrm{r}}\left(t_{\mathrm{a}}\right) \mathbf{M}^{\mathrm{T}},
\end{aligned}
$$

where $\langle$.$\rangle is the expectation operator. The ET KF predicted wind signal variance$ $\boldsymbol{s}_{\mathrm{ETKF}}^{q}\left(t_{\mathrm{a}}\right)$ at the future analysis (targeting) time, given by the diagonal terms of $\mathbf{S}^{q}\left(t_{\mathrm{a}}\right)$, averaged over the 850,500 and $200 \mathrm{mb}$ levels, using a 36-hour-old ECMWF ensemble (Molteni et al. 1996; Buizza et al. 1998), and the square of the NCEP signal realization $s_{\mathrm{NCEP}}^{q 2}\left(t_{\mathrm{a}}\right)$ produced using the operational 3D-Var scheme, are shown in Figs. 2(a) and (b), respectively, for targeted dropwindsondes released by an aircraft en route from Honolulu to Anchorage on 5 February 1998. The ET KF signal variance and square of the NCEP signal are clearly different in structure. Although there is no reason why a signal variance should look exactly like an individual squared signal realization, as we describe later, differing error covariance assumptions mean that ET KF signals and signal variance would be systematically different from NCEP signals and signal variance.

The Kalman-filter estimate of the analysis (or forecast) error covariance matrix $\mathbf{P}^{q}(t)$ pertaining to the $q$ th combined network of routine plus targeted observations is given by

$$
\mathbf{P}^{q}(t)=\mathbf{P}^{\mathrm{r}}(t)-\mathbf{S}^{q}(t),
$$

as derived in Bishop et al. (2001). The signal covariance matrix is therefore precisely the reduction in analysis (forecast for $t>t_{\mathrm{a}}$ ) error covariance due to the $q$ th set of targeted observations, compared with the routine-analysis (forecast) error covariance. This is only strictly true if errors evolve linearly and the routine and observationalerror covariances prescribed in the data-assimilation scheme are accurate. However, error covariances assumed by NCEP's currently operational 3D-Var assimilation scheme (Parrish and Derber 1992) and by the ET KF are very different, and both sets of covariances are inaccurate. The observational-error covariance matrix $\widehat{\mathbf{R}}^{q}$ assumed by the ET KF is presently diagonal with no spatial variation in the error variances of the horizontal wind component at each of the three levels. This simplified matrix is based upon the NCEP observational errors described in Wu and Joo (1996). The corresponding observation-error statistics used in NCEP's data-assimilation scheme vary with the many types of observations that are available, and the observation location.

The routine-analysis error covariance matrix that is consistent with the assumptions of the NCEP 3D-Var scheme can be written in the form

$$
\mathbf{P}_{\mathrm{NCEP}}^{\mathrm{r}}\left(t_{\mathrm{a}}\right)=\mathbf{B}-\mathbf{B} \widehat{\mathbf{H}}_{\mathrm{a}}^{\mathrm{r}} \mathrm{T}\left(\widehat{\mathbf{H}}_{\mathrm{a}}^{\mathrm{r}} \mathbf{B} \widehat{\mathbf{H}}_{\mathrm{a}}^{\mathrm{r} \mathrm{T}}+\widehat{\mathbf{R}}^{\mathrm{r}}\right)^{-1} \widehat{\mathbf{H}}_{\mathrm{a}}^{\mathrm{r}} \mathbf{B},
$$


(a) ETKF : $98020500+00 \mathrm{~h}$

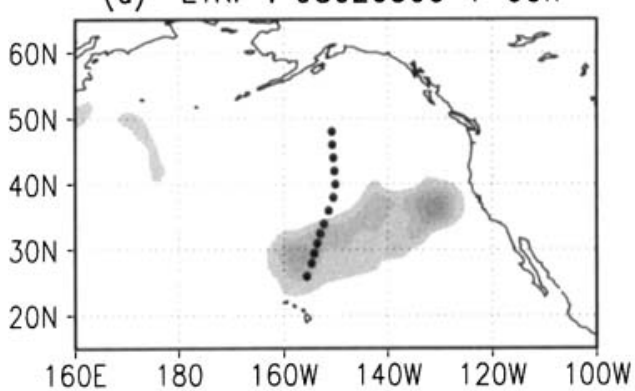

(c) ETKF : $98020500+12 \mathrm{~h}$

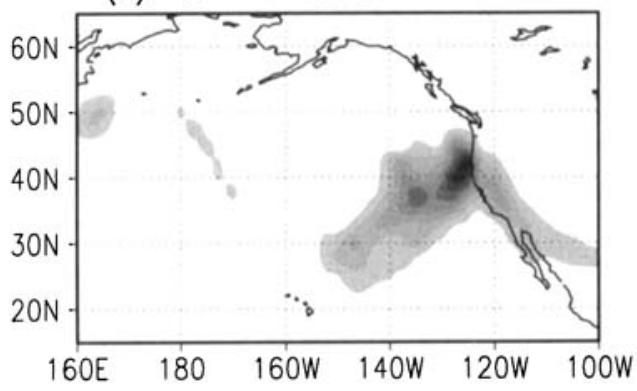

(e) ETKF : $98020500+24 \mathrm{~h}$

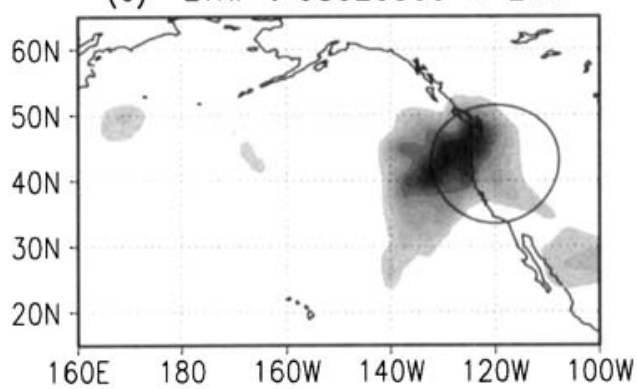

(g) ETKF : $98020500+36 \mathrm{~h}$

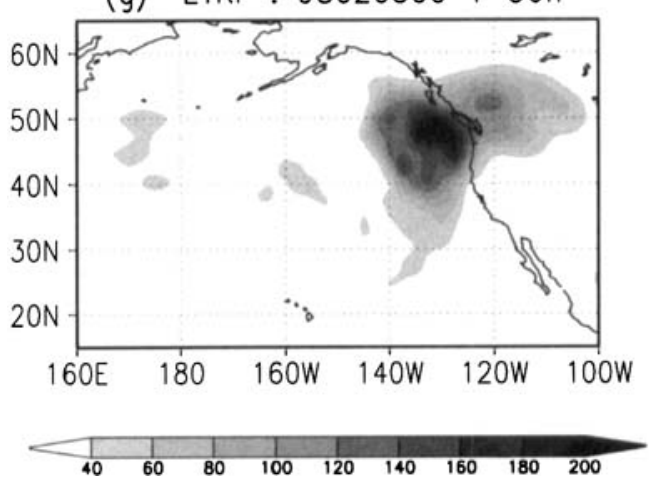

(b) NCEP : $98020500+00 \mathrm{~h}$

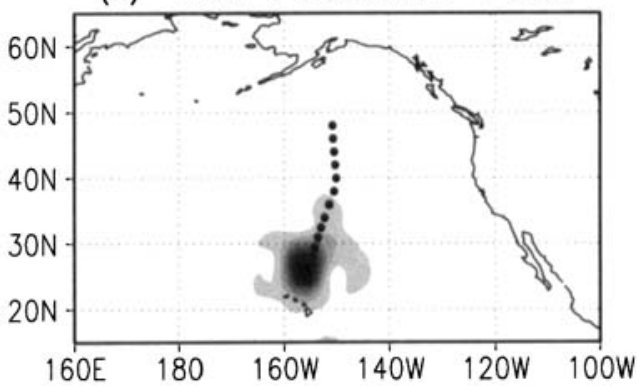

(d) NCEP : $98020500+12 \mathrm{~h}$

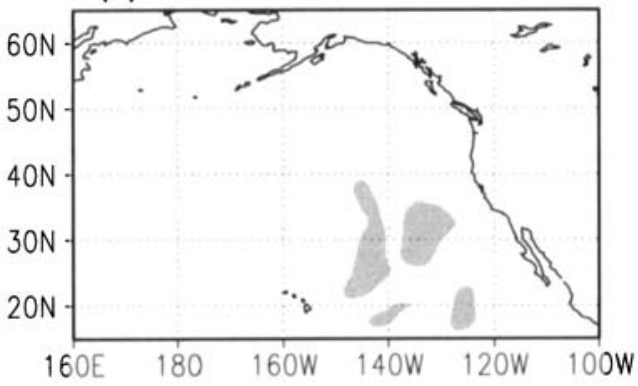

(f) NCEP : $98020500+24 \mathrm{~h}$

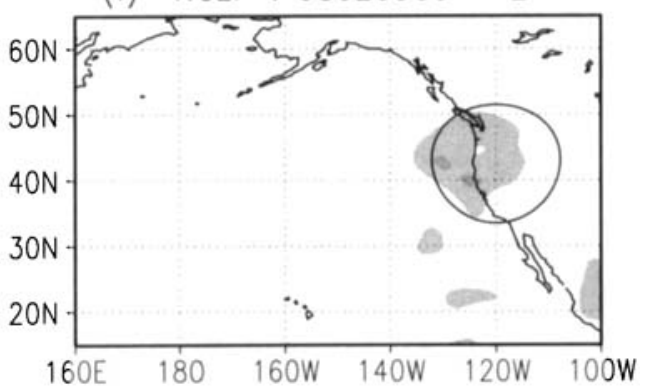

(h) NCEP : $98020500+36 \mathrm{~h}$
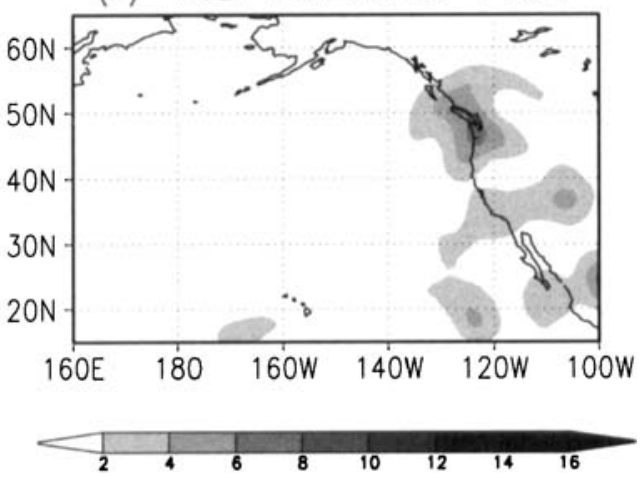

Figure 2. (a), (c), (e) and (g) ET KF predicted wind signal variance at $+00,+12,+24$ (verification) and $+36 \mathrm{~h}$, respectively, after the analysis time $t_{\mathrm{a}}=00$ UTC 5 February 1998, based on a 50-member ECMWF ensemble initialized at 12 UTC 3 February 1998. (b), (d), (f) and (h) Squared NCEP MRF wind signal produced at 00 UTC 5 February 1998 , for analyses at $+00 \mathrm{~h}$, and forecasts valid $+12,+24$ and $+36 \mathrm{~h}$, respectively, after this time. Units are $\mathrm{m}^{2} \mathrm{~s}^{-2}$. The dots in (a) and (b) mark the positions of the dropwindsonde observation points. The circles in (e) and (f) mark the forecast verification region. 
where $B$ is the quasi-isotropic error covariance matrix assumed by the NCEP 3D-Var scheme and $\widehat{\mathbf{H}}_{\mathrm{a}}^{\mathrm{r}}$ is the observation operator corresponding to the routine observational network. The B matrix does not account for spatial or temporal variations of error.

The routine-analysis error covariance matrix used by the ET KF is expressed as the outer product of the matrix of transformed ensemble perturbations $\mathbf{Z}^{\mathrm{r}}\left(t_{\mathrm{a}}\right)$,

$$
\mathbf{P}_{\mathrm{ETKF}}^{\mathrm{r}}\left(t_{\mathrm{a}}\right)=\mathbf{Z}^{\mathrm{r}}\left(t_{\mathrm{a}}\right) \mathbf{Z}^{\mathrm{rT}}\left(t_{\mathrm{a}}\right) \text {. }
$$

The ensemble perturbations have been transformed in a manner that makes them appropriate for a routine observational network at time $t_{\mathrm{a}}$. Further details of the transformation and its limitations are given in section 2(i) of MEA. The ensemble-based specification of the background-error covariance matrix as an outer product of the matrix of ensemble perturbations (as in Eq. 5) is inaccurate for several reasons. One major reason is that the limited number of available ensemble forecasts leads to a rank-deficient estimate of the background-error covariance matrix. This implies that spurious correlations may exist between state variables at large distances. Additionally, the accuracy of the backgrounderror covariance matrix is dependent on the quality of the ensemble forecast. Another reason that this matrix (and hence the ET KF signal variance) may be inaccurate is that the ensemble members generated at least 36 hours prior to time $t_{\mathrm{a}}$ would not account for any targeted observations that are taken 24 hours prior to $t_{\mathrm{a}}$, whereas an NCEP signal produced at $t_{\mathrm{a}}$ would depend on the effects of these targeted observations.

Equations (4) and (5) give very different specifications of the routine-analysis error covariance matrix $\mathbf{P}^{\mathrm{r}}\left(t_{\mathrm{a}}\right)$, both of which are inaccurate. Since the signal covariance estimate is highly dependent on $\mathbf{P}^{\mathrm{r}}\left(t_{\mathrm{a}}\right)$ as specified in the data-assimilation scheme (from Eq. (2)), a signal produced by an ET KF data-assimilation scheme would therefore be likely to differ significantly in structure from a signal produced by 3D-Var.

\section{(b) Evolution of ET KF signal variances versus NCEP signal realizations}

To illustrate potential similarities and differences between evolving ET KF and NCEP signal structures, we examine the 5 February 1998 flight that took place during the NORPEX* field experiment (Langland et al. 1999; Szunyogh et al. 1999b). At the future analysis time, the ET KF signal variance $\delta_{\mathrm{ETKF}}^{q}\left(t_{\mathrm{a}}\right)$ (Fig. 2(a)) asserts that errors at the observational locations are highly correlated with the elongated structure of a frontal zone. In contrast, the squared NCEP signal realization $s_{\mathrm{NCEP}}^{q 2}\left(t_{\mathrm{a}}\right)$ produced by the operational 3D-Var scheme (Fig. 2(b)) is localized near the observation sites with no indication of the existing frontal structure. The major differences between signals produced by the ET KF and 3D-Var schemes follow from the difference between routine-analysis error covariance matrices that would be produced by Eqs. (4) and (5), respectively. In general, the ET KF signal produced by an observation will spread along a coherent dynamical feature such as a front or a tropical cyclone, whereas the 3D-Var scheme is more likely to produce signals localized near observation sites. Nevertheless, one can encourage the 3D-Var signal to conform more closely to the predicted ET KF signal by taking observations in atmospheric regions where the ET KF signal variance is locally large at the future analysis time (see MEA for more details).

The ET KF suggests that differences between the routine-analysis estimate and the observations north of $35^{\circ} \mathrm{N}$ are likely to be minimal, and hence any signal within this region also ought to be small (Fig. 2(a)). Indeed, the squared NCEP signal has small amplitude north of $35^{\circ} \mathrm{N}$, despite the fact that closely spaced observations extend to $50^{\circ} \mathrm{N}$ (Fig. 2(b)). The ET KF therefore asserted correctly in this case that a large signal

* North Pacific Experiment. 
north of $35^{\circ} \mathrm{N}$ was unlikely. This is not to say that observations north of $35^{\circ} \mathrm{N}$ are useless. Such observational locations may be in areas where structures, while initially very small, may grow rapidly and propagate into the verification region.

The evolved ET KF signal variance and squared NCEP signal at $+12,+24$ and $+36 \mathrm{~h}$ after the analysis time $t_{\mathrm{a}}$ are shown alongside each other in Figs. 2(c), (e), (g) and (d), (f), (h), respectively. The ET KF signal variance is calculated using a linear transformation of evolved ECMWF ensemble perturbations (initialized $36 \mathrm{~h}$ prior to $t_{\mathrm{a}}$ ), and the NCEP signal is produced from parallel NCEP Medium-Range Forecast (MRF) model runs initialized at $t_{\mathrm{a}}$ with and without the targeted observations. The NCEP signal decays sharply in the first $12 \mathrm{~h}$ (Fig. 2(d)), which may be a result of the signal failing to project onto growing structures. Such structures are often highly baroclinic, and a 3D-Var scheme may not be able to resolve these structures with a limited number of observations. Furthermore, since the ensemble perturbations are designed to sample the subspace of expanding atmospheric structures, they generally better represent the growing than the decaying and neutral structures. However, analysis increments produced in operations are not likely to ignore neutral or decaying structures. Similar results obtained during FASTEX and a more detailed discussion of this point are given in Szunyogh et al. (1999a). Despite the structural differences between the ET KF signal variance and NCEP signal at $+12 \mathrm{~h}$, a qualitative correspondence is evident both at +24 and $+36 \mathrm{~h}$ (although the ET KF signal variance maximum amplifies and shifts slightly towards the north-west at $+36 \mathrm{~h}$ ). Both signals are maximal within the verification region at the verification time ( +24 h, Figs. 2(e) and (f)). As discussed in Szunyogh et al. (1999a,b) and Toth et al. (2001), the propagation of the NCEP signal is an important diagnostic in evaluating the efficacy of targeted observations. Ideally, part of the signal from the targeted dropwindsondes would always propagate into the verification region at the verification time. If the operational model and data-assimilation scheme behaved adequately, this signal propagation should, on average, translate into a reduction in forecast-error variance. However, higher inaccuracies in error covariances specified by the data-assimilation scheme can lead to increased forecast-error variance (Bishop 2001, personal communication). To rectify difficulties in error covariance specification in current data-assimilation schemes, substantial effort is being expended to produce improved estimates of the background-error covariance matrix (Evensen 1994; Evensen and van Leeuwen 1996; Houtekamer and Mitchell 1998; Burgers et al. 1998; Mitchell and Houtekamer 2000; Anderson and Anderson 1999; Janisková et al. 1999; Rabier et al. 2000; Hamill and Snyder 2000; Bishop et al. 2001).

\section{STATISTICAL CORRECTION OF ET KF SIGNAL VARIANCE}

During the 2000 Winter Storm Reconnaissance Program (WSR00), the optimal flight track was selected from 42 pre-designed tracks out of Anchorage and Honolulu (see MEA; Toth et al. 2001)*. In this section, we test whether the ET KF can be used to make quantitative predictions of operational signal variance at both the future analysis and verification times, by seeking a statistical relationship between the respective signal variances. If a linear, increasing relationship exists, the difference between the ET KF and NCEP signal variance magnitudes could be resolved by rescaling the ET KF signal variance by a simple factor.

* A combined ensemble of 25 ECMWF members (initialized 36 hours prior to time $t_{\mathrm{a}}$ ), 2 NCEP MRF members ( $36 \mathrm{~h}$ ), and 5 NCEP MRF members (24 h) (Toth and Kalnay 1997) was used in these calculations. 

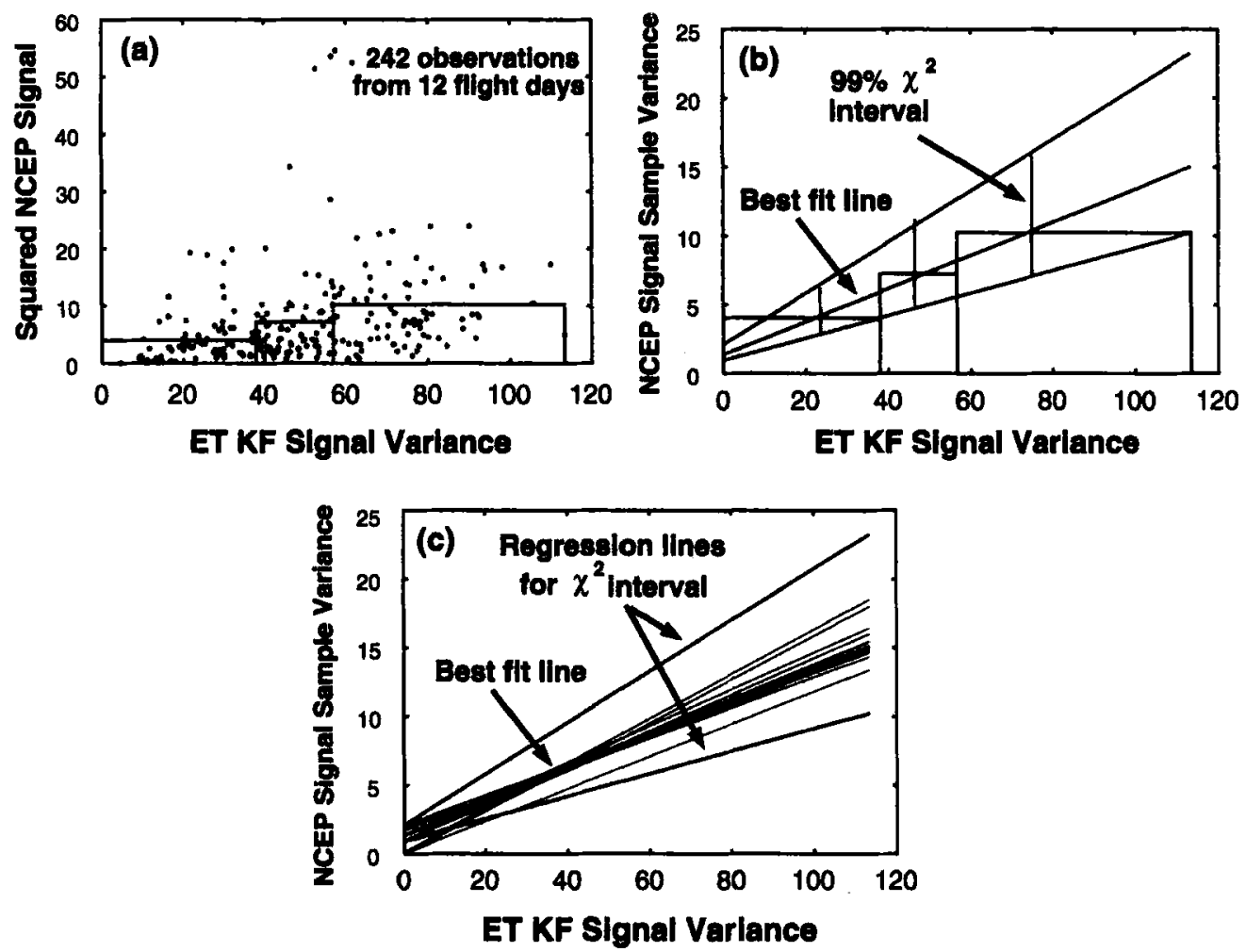

Figure 3. (a) Scatter plot of squared NCEP signal and ET KF signal variance at the analysis time $t_{2}$, for 242 WSR00 observation sites. The points are divided into three categories of 81, 81 and 80, arranged in order of increasing ET KF signal variance. The height of each of the three blocks gives the NCEP sample signal variance of that category. (b) Error bars represent the $99 \% \chi^{2}$ confidence interval of the NCEP signal variance for each of the three categories, plotted at the position of mean ET KF signal variance for that category. The three sloping lines represent the best-fit regression line between ET KF and NCEP signal variances, and corresponding lines for the $99 \%$ confidence limits. (c) Thin lines: best-fit lines calculated in a similar manner to (b), but with data from each of the 12 flight days suppressed; thick lines: the regression lines of (b) (in which no data were denied) are shown for comparison.

\section{(a) Future analysis (targeting) time $t_{\mathrm{a}}$}

We first evaluate the relationship between ET KF and NCEP wind signal variances (over the 850, 500 and $200 \mathrm{mb}$ levels) using all 242 dropwindsonde locations from the 12 flight days between 23 January and 16 February 2000 as our sample. The squared NCEP signal $s_{\mathrm{NCEP}}^{q 2}\left(t_{\mathrm{a}}\right)$ and the predicted ET KF signal variance $s_{\mathrm{ETKF}}^{q}\left(t_{\mathrm{a}}\right)$ at each location are plotted in Fig. 3(a). A small ET KF signal variance at a location is expected to indicate that any realization of the squared NCEP signal at the same site is small. If $s_{\mathrm{NCEP}}^{q 2}\left(t_{\mathrm{a}}\right)$ were instead large, the ET KF would likely have failed since the squared signal lay several standard deviations away from its expected mean value (i.e. the signal variance). The converse is not necessarily true. A large signal variance suggests that the distribution of signals is broader than if it were small. However, if signals were normally distributed, then its most likely value would be zero even if the variance were large. Thus, a high ET KF signal variance does not automatically imply that an NCEP signal ought to be large. A quick inspection of Fig. 3(a) shows several observational locations where high predicted ET KF signal variance corresponds to an NCEP signal of large magnitude, and also some locations in which the NCEP signal is small. 
Since only one realization of the squared NCEP signal $s_{\mathrm{NCEP}}^{q 2}\left(t_{\mathrm{a}}\right)$ exists for each of the 242 observations, our sample sizes are small. We calculate sample variances $\delta_{\text {NCEP }}^{q}\left(t_{\mathrm{a}}\right)$ of the NCEP signal in Fig. 3(a) by placing signal realizations in order of increasing ET KF signal variance into three groups. The first, second and third groups contain the lowest 81 , middle 81 and highest 80 values of ET KF signal variance, respectively. The NCEP sample signal variance $s_{\mathrm{NCEP}}^{q}\left(t_{\mathrm{a}}\right)$ is the average of all squared NCEP signals $s_{\text {NCEP }}^{q_{2}}\left(t_{\mathrm{a}}\right)$ in that sample. Each horizontal bar on Fig. 3(a) gives the NCEP signal variance for the corresponding sample. These values increase monotonically with respect to the average value of the ET KF signal variance in each group.

To test statistically whether the ET KF and NCEP signal variances are related, we first assume that all signals are independent and normally distributed, so the signal variance comes from a $\chi^{2}$ distribution with 80 degrees of freedom (79 in the third group). The $99 \%$ confidence interval for the NCEP signal variance of each sample is then plotted in Fig. 3(b) against the average ET KF signal variance in each group. Within these confidence intervals, it is impossible to draw a straight line in which the NCEP signal variance is a decreasing function of the ET KF signal variance. Based on our limited samples, the probability of this occurring is therefore tiny (less than $\left.(0.005)^{2}\right)$. The best-fit lines for the linear relationship between the ET KF and NCEP signal variances, and for the upper and lower confidence limits, are also shown in Fig. 3(b). The gradient of the best-fit line is roughly $1 / 8$. This line passes close to, although not directly through, the origin. Nevertheless, a linear statistical rescaling using this factor can enable the ET KF to give approximate predictions of the NCEP signal variance at the analysis time $t_{\mathrm{a}}$.

A second test of the robustness of the relationship between the ET KF and NCEP signal variances is performed by eliminating data from each of the 12 flight days, and producing best-fit lines similar to those shown in Fig. 3(b). In this manner, the variability of the ET KF-NCEP signal variance relationship with respect to the withdrawal of independent sub-samples from each flight day is shown. This test is based upon a type of bootstrap method called the jackknife (Efron 1982). The 12 best-fit lines in Fig. 3(c) all lie within the regression lines for the $99 \%$ confidence interval of NCEP signal variance, except at low values. The general similarity of these 12 lines leads us to conclude that the relationship between the ET KF and NCEP signal variances is fairly stable if data at observation locations are added or removed.

Although the two tests described above confirmed some qualitative similarities, the ET KF signal variance overestimated the NCEP signal magnitude by nearly an order of magnitude. Several possible reasons for this discrepancy exist. Most crucially, the ET KF's overestimation of signal variance is a strong indication that our estimate of the routine-analysis error covariance matrix $\mathbf{P}^{\mathrm{r}}\left(t_{\mathrm{a}}\right)$ is leading to local estimates of analysis error that are unrealistically large. Our constraint that the ensemble perturbations have, in a globally averaged sense, a sample variance equal to the average analysis-error variance appears to be inappropriate. Work is under way to improve our estimate of the routine-analysis error covariance matrix.

The ability of the ET KF to predict NCEP signal variance has potentially significant implications in operational data analysis. Data quality-control schemes often use estimates of the root-mean-square (r.m.s.) difference between the background and observation to decide whether or not to reject observations. More specifically, if the difference between the observation and background exceeds the r.m.s. difference by some preset factor, the observation is rejected on the grounds that the observation is probably faulty in some way. Our results confirm the expectation that the r.m.s. difference between background and observation is a flow-dependent quantity. For example, in frontal regions one 
would expect it to be larger than it would be in the centre of a high-pressure system. Our results indicate that quality-control schemes could be improved by using the ET KF in conjunction with a plot like Fig. 3(b) to predict the flow-dependent r.m.s. difference between background and observation rather than a flow-independent estimate.

\section{(b) Forecast verification time $t_{\mathrm{f}}$}

The ability of the ET KF to predict signal variance within the pre-selected verification region at the future verification time is now assessed. This ability may be compromised by several factors. Firstly, as shown in Figs. 2(b) and (d), the NCEP signal may decay much more rapidly than the signal variance anticipated by the ET KF after the observation time. The quasi-isotropic 3D-Var data-assimilation scheme has limited ability in producing signals that project onto growing, baroclinic structures located away from the observation sites. A decay in the signal is often evident 12 hours after the observations are taken (see Figs. 2(b) and (d) for example, and also Szunyogh et al. 1999a). In contrast, the ET KF produces signal variance structures that usually amplify as they propagate downstream (since ensemble forecasts attempt to capture growing structures). Secondly, the ET KF uses combined ECMWF and NCEP ensemble forecasts initialized 24-36 $\mathrm{h}$ prior to the analysis time $t_{\mathrm{a}}$ to predict signal variance, whereas the NCEP signal is produced using NCEP MRF forecasts initialized at $t_{\mathrm{a}}$. Thirdly, the ET KF uses a linear theory (although the ensemble perturbations are nonlinear). Each of these factors suggest that the correspondence between ET KF and NCEP signal variance may be less clear at the verification time $t_{\mathrm{f}}$ than at the earlier analysis time $t_{\mathrm{a}}$.

To evaluate the relationship between ET KF and NCEP signal variance at the verification time, the ET KF forecast signal variance $s_{\mathrm{ETKF}}^{q}\left(t_{\mathrm{f}}\right)$ and squared NCEP forecast signal $s_{\operatorname{NCEP}}{ }_{2}\left(t_{f}\right)$ is calculated at every grid point (2.5 2 resolution) within each verification region and plotted in Fig. 4(a), for 30 potentially important weather events during the WSR00 period. These 30 cases were selected in real time, based upon the potential for significant precipitation, and a relatively high uncertainty in the forecast. The scatter plot in Fig. 4(a) shows that high NCEP signals often correspond to larger predicted ET KF signal variances. Crucially, there are no anomalous points in which the ET KF predicted a low signal variance and the NCEP signal realization turned out to be of high magnitude.

In a similar manner to Fig. 3(b), the points are placed into four groups of 389 and one of 388, depending on their ET KF signal variance value (Fig. 4(b)). The NCEP sample signal variance (average of squared signals) $s_{\mathrm{NCEP}}^{q}\left(t_{\mathrm{f}}\right)$ increases with average ET KF signal variance in each group, as shown by the horizontal bars on Fig. 4(b). Assuming that all signal realizations are independent and normally distributed, the $99 \%$ $\chi^{2}$ confidence limits of NCEP signal variance for each sample show that the probability of a non-monotonic linear relationship between the ET KF and NCEP signal variances is minimal. Assuming that the relationship is linear, the ET KF signal variance ought to be reduced by a factor of roughly 14 to give a scale comparable to the NCEP forecast signal variance. The fact that this scaling factor is higher than the corresponding factor of 8 at the analysis time demonstrates that the growth rate of the operational NCEP signal is, on average, smaller than that predicted by the ET KF.

As was performed in section 3(a), we also analyse the uncertainty in our estimate of the rescaling factor in the ET KF-NCEP signal variance relationship due to subsampling. To do this, we remove a dataset corresponding to one of the 30 verification regions, and recalculate the best-fit line in the same manner as in Fig. 4(b). We do this for all verification datasets. The 30 best-fit lines in Fig. 4(c) illustrate that the variability 

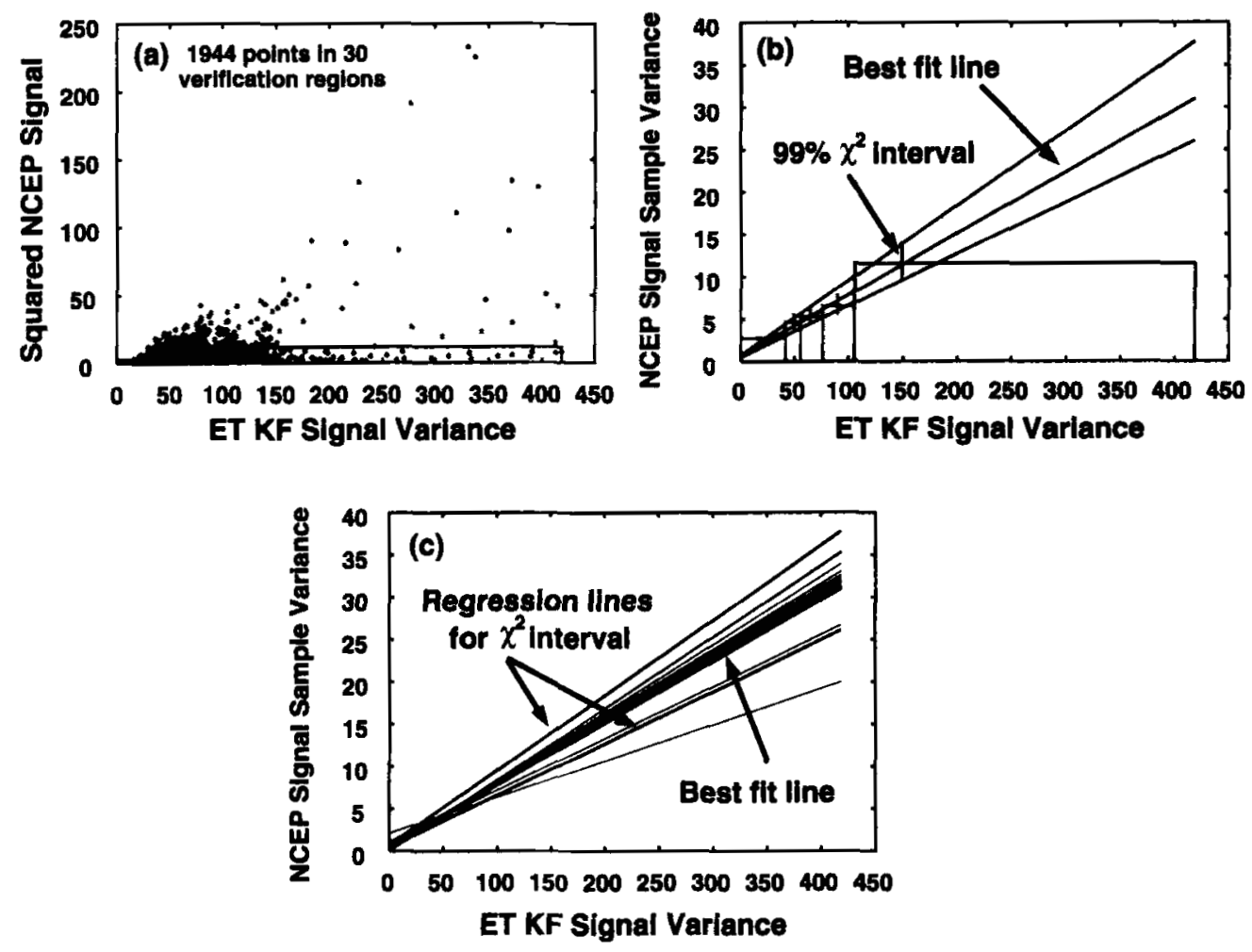

Figure 4. (a) Scatter plot of squared NCEP forecast signal versus ET KF forecast-signal variance, for all 1944 grid points within 30 WSR00 verification regions. The points are divided into four categories of 389 and one of 388, arranged in order of increasing ET KF signal variance. The height of each of the five blocks gives the NCEP sample signal variance of that category. (b) Error bars represent the $99 \%$ confidence interval of the NCEP signal variance for each of the five categories, plotted versus the mean ET KF signal variance for that category. The three lines represent the best-fit regression line between ET KF and NCEP signal variances, and corresponding lines for the $99 \%$ confidence limits. (c) Thin lines: best-fit lines calculated in a similar manner to (b), but with data from each of the 30 verification regions denied; thick lines: regression lines of (b) (in which no data were denied).

is usually fairly small; 29 of these lines lie within the regression lines of the $99 \% \chi^{2}$ confidence limits of Fig. 4(b). Although one case (in which the ET KF signal variance and NCEP signal magnitudes were unusually high) did alter the gradient of the best-fit line significantly, the linear relationship between ET KF and NCEP signal variance at the verification time was generally found to be robust.

\section{USING THE STATISTICAL RESCALING TO ESTIMATE THE REDUCTION IN NCEP FORECAST-ERROR VARIANCE}

Now that a linear map between ET KF and NCEP forecast-signal variances has been established for our sample, the next step is to test the relationship between the NCEP forecast-signal variance $s_{\mathrm{NCEP}}^{q}\left(t_{\mathrm{f}}\right)$ and the reduction in NCEP forecast-error variance $\mathcal{R}_{\mathrm{NCEP}}^{q}\left(t_{\mathrm{f}}\right)$ due to the targeted observations. This is done using the two parallel NCEP analysis-forecast cycles, which, respectively, include and exclude the targeted observations in their assimilation cycles. If the relationship is found to be monotonic increasing, the ET KF can be used, via the statistical rescaling factor derived in subsection 3(b), to make quantitative predictions of the effect of targeted observations on NCEP forecast-error variance. 
The NCEP forecast error $\epsilon_{\mathrm{NCEP}}\left(t_{\mathrm{f}}\right)$ at a verification location is approximated by the difference between the forecast $x_{\mathrm{NCEP}}\left(t_{\mathrm{f}} \mid \mathbf{H}_{\mathrm{a}}\right)$ conditional on NCEP'S observation operator $\mathbf{H}_{\mathrm{a}}$ at the analysis time $t_{\mathrm{a}}$, and the NCEP analysis $x_{\mathrm{NCEP}}\left(t_{\mathrm{f}} \mid \widehat{\mathbf{H}}_{\mathrm{f}}^{\mathrm{t}}\right)$ produced using the observation operator $\widehat{\mathbf{H}}_{\mathrm{f}}^{\mathrm{r}}$ corresponding to the routine observational network at verification time $t_{\mathrm{f}}$. We expect that the analysis, while never perfect, is reasonable in regions over land where the observational network is augmented by rawinsonde balloon and ground-based observations. Using an analysis rather than observational data to estimate the truth also gives a much larger sample of verification locations for our study. The respective errors of NCEP forecasts initialized with routine (operator $\widehat{\mathbf{H}}_{\mathbf{a}}^{\mathbf{r}}$ ) and routine-plus-targeted (operator $\mathbf{H}_{\mathrm{a}}^{q}$ ) observational networks at time $t_{\mathrm{a}}$ are then given by

$$
\epsilon_{\mathrm{NCEP}}^{\mathrm{r}}\left(t_{\mathrm{f}}\right)=x_{\mathrm{NCEP}}\left(t_{\mathrm{f}} \mid \widehat{\mathbf{H}}_{\mathrm{a}}^{\mathrm{r}}\right)-x_{\mathrm{NCEP}}\left(t_{\mathrm{f}} \mid \widehat{\mathrm{H}}_{\mathrm{f}}^{\mathrm{r}}\right)
$$

and

$$
\epsilon_{\mathrm{NCEP}}^{q}\left(t_{\mathrm{f}}\right)=x_{\mathrm{NCEP}}\left(t_{\mathrm{f}} \mid \mathbf{H}_{a}^{q}\right)-x_{\mathrm{NCEP}}\left(t_{\mathrm{f}} \mid \widehat{\mathbf{H}}_{\mathrm{f}}^{\mathrm{r}}\right),
$$

respectively. Figures $5(\mathrm{a})$ and (c) show the respective squared values of forecast error $\epsilon_{\mathrm{NCEP}}^{r 2}\left(t_{\mathrm{f}}\right)$ and $\epsilon_{\mathrm{NCEP}}^{q 2}\left(t_{\mathrm{f}}\right)$ at all 1944 grid points within the 30 WSR00 verification regions, versus the squared NCEP signal $s_{\mathrm{NCEP}}^{q^{2}}\left(t_{\mathrm{f}}\right)$ at the corresponding locations. The NCEP forecast-error variance for each sample of 389 (388 in the fifth group) is then calculated as the average of the realizations of squared forecast error within each sample (Figs. 5(b) and (d)). The sample variance is lower in each group in Fig. 5(d) compared with Fig. 5(b), which suggests that the targeted observations did, on average, reduce the forecast error within the WSR00 verification regions. However, if we examine the reduction in squared NCEP forecast error due to the targeted observations at each verification point

$$
\rho_{\mathrm{NCEP}}^{q}\left(t_{\mathrm{f}}\right)=\epsilon_{\mathrm{NCEP}}^{\mathrm{r} 2}\left(t_{\mathrm{f}}\right)-\epsilon_{\mathrm{NCEP}}^{q 2}\left(t_{\mathrm{f}}\right)
$$

in Fig. 5(e), we notice a significant number (37.6\% of the 1944 points) of forecast degradations (where the ordinate is less than zero). These degradations may occur, partly as a result of a data-assimilation scheme that does not optimally exploit the information contained in the dropwindsonde observations, and also because of model error. One should also note that forecast degradations may occur even if a data-assimilation scheme is perfect, but the chances of significant degradations are higher if error covariances are specified less accurately in the data-assimilation scheme.

Averaging the squared NCEP signals in each of the five groups to give the NCEP sample signal variance $s_{\mathrm{NCEP}}^{q}\left(t_{\mathrm{f}}\right)$, and calculating the reduction in NCEP forecast-error variance $\mathscr{R}_{\mathrm{NCEP}}^{q}\left(t_{\mathrm{f}}\right)$ by averaging over all realizations of $\rho_{\mathrm{NCEP}}^{q}\left(t_{\mathrm{f}}\right)$ in each group, we find a strictly monotonic increasing relationship between the NCEP signal variance and reduction in forecast-error variance (Fig. 5(f)). This is strongly supported by the $99 \%$ $\chi^{2}$ confidence intervals for reduction in forecast-error variance. If errors grew linearly and error covariances were specified accurately, one would expect this relationship to be linear and of unit gradient (from Eq. (3)). Since a linear relationship could not be established here, we fit a cubic spline, using zero second derivatives at the end-points, to give an indication of the relationship between NCEP signal variance and reduction in forecast-error variance.

The uncertainty in this relationship due to removing independent sub-samples from the full sample is shown in Fig. 6, using the same technique as in Fig. 4(c). Although 

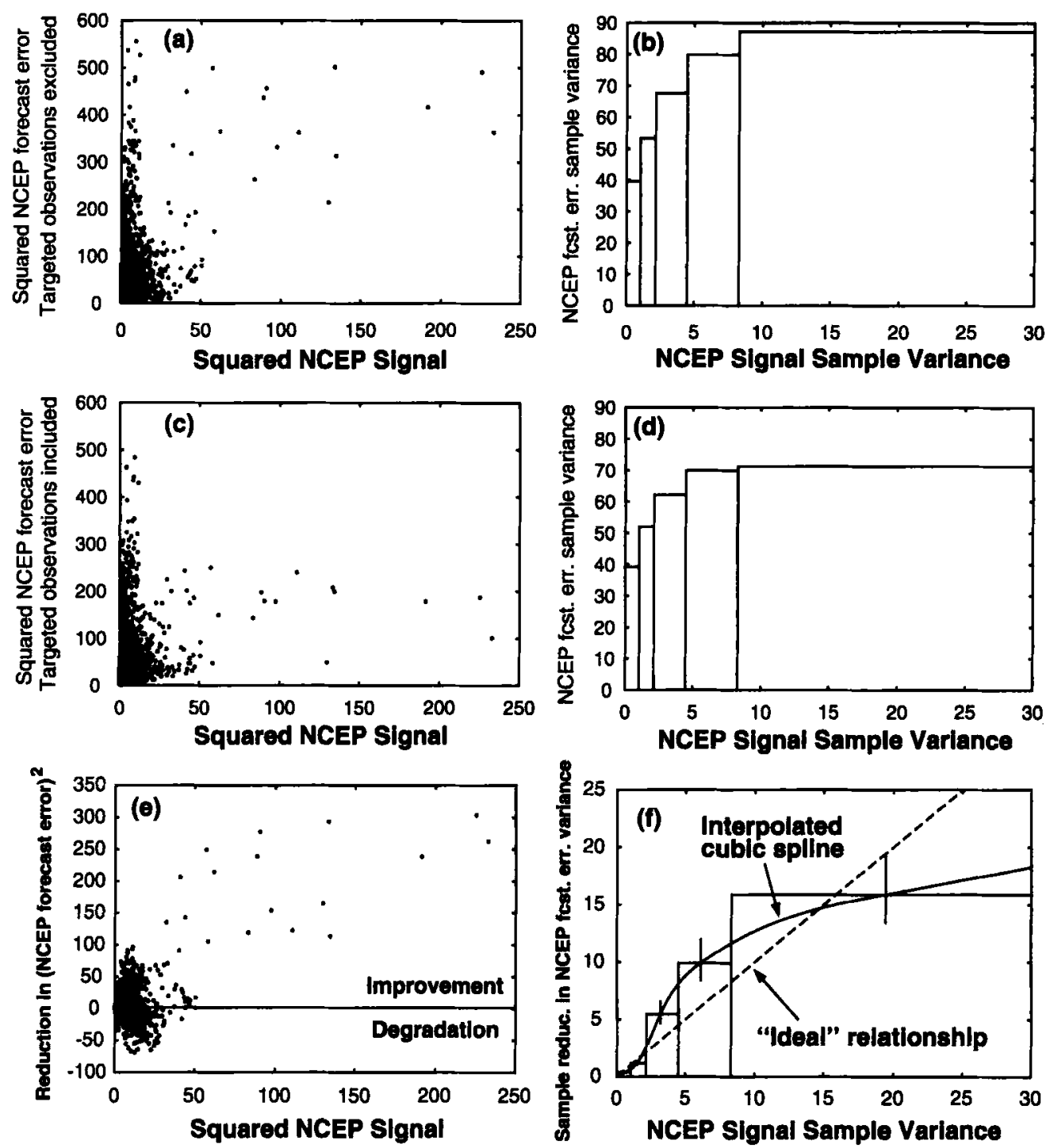

Figure 5. (a) Scatter plot of squared forecast error for NCEP MRF forecasts that excluded targeted observations, versus squared NCEP signal, for all 1944 grid points within the 30 verification regions chosen during WSR00. (b) All points on the scatter plot in (a) are grouped into four categories of 389 and one of 388, arranged in order of increasing squared NCEP signal. The heights of each of the five blocks give the forecast-error sample variance, for NCEP MRF forecasts that excluded targeted observations. (c) As in (a), but the ordinate represents squared forecast error for NCEP MRF forecasts that included targeted observations. (d) Using the same method as in (b), the heights of each block gives the forecast-error sample variance, for NCEP MRF forecasts that included targeted observations. (e) Scatter plot of reduction in squared NCEP forecast error due to the targeted observations versus squared NCEP signal (i.e. the difference between squared forecast errors plotted in (a) and (c)). (f) The height of each block corresponds to the reduction in NCEP forecast-error variance in each sample (i.e. the difference between sample variances in (b) and (d)). A cubic spline is drawn through the respective sample variances. The 'ideal' relationship, where the reduction in forecast-error variance is identical to the signal variance, is also shown for comparison. 


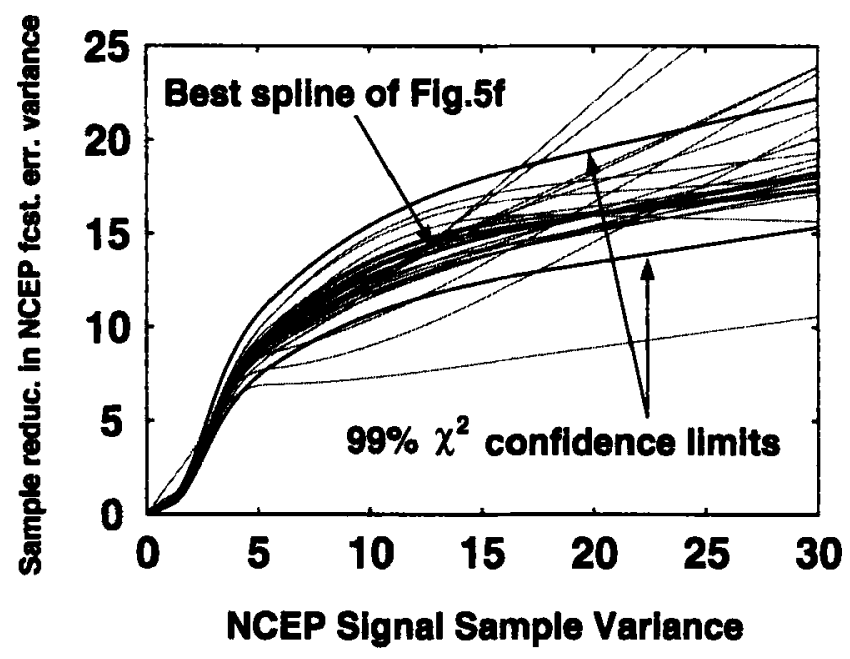

Figure 6. Thin lines: cubic splines calculated in a similar manner to Fig. 5(f), but with data from each of the 30 verification regions suppressed. Thick lines the best-fit cubic spline and the splines that pass through the $99 \%$ $\chi^{2}$ confidence limits of Fig. $5(f)$ are shown for comparison.

the majority of the 30 cubic splines lie within the splines drawn through the $99 \% \chi^{2}$ confidence limits, there are a few outliers. However, these mostly occur at high values of the NCEP signal variance $\delta_{\text {NCEP }}^{q}\left(t_{\mathrm{f}}\right)$, and we only have one data point in the spline interpolation for $s_{\mathrm{NCEP}}^{q}\left(t_{\mathrm{f}}\right)>10$. In general, the relationship is not highly sensitive to the removal of independent verification cases.

The tests illustrated in Figs. 5 and 6 imply that there is a strong, monotonic increasing relationship between the NCEP reduction in forecast-error variance and the NCEP signal variance. Via the rescaling factor calculated in Fig. 4(b), a relationship between the reduction in NCEP forecast-error variance and the ET KF prediction of signal variance can be deduced. Combining the factor of approximately 14 for the bestfit line of Fig. 4(b) with the best spline of Fig. 5(f), the new optimal relationship is shown (Fig. 7(a)). Using the $99 \% \chi^{2}$ confidence limits for both the rescaling factors of Fig. 4(b) and the cubic splines of Fig. 5(f), new confidence limits are produced. While the error bounds in this relationship are fairly large, the potential of using the ET KF to predict the reduction in NCEP forecast-error variance is demonstrated. By systematically removing data from each of the 30 WSR00 verification regions and combining the appropriate rescaling factors (Fig. 4(c)) and cubic splines (Fig. 6) for each dataset, we observe that most of the 30 new curves in Fig. 7(a) lie within the 99\% confidence limits. Hence, the relationship between ET KF signal variance and reduction in NCEP forecast-error variance is not greatly sensitive to either the addition or removal of new data.

If enough data from targeted observations were available and improvements were made to the ensemble forecasts, the ET KF targeting strategy, and the operational analysis-forecast cycle, a more robust relationship between the estimated ET KF signal variance and reduction in NCEP forecast-error variance could be formally established. As an illustration, the rescaling factor of approximately 14 is used in Fig. 7(b) to produce physically meaningful values of the ET KF signal variance. These values could in turn be translated into meteorological or economic estimates of the likelihood of forecast improvements for any future deployment of targeted observations (for example, see Katz and Murphy (1997) and references therein for discussions on the economic value of weather forecasts). 

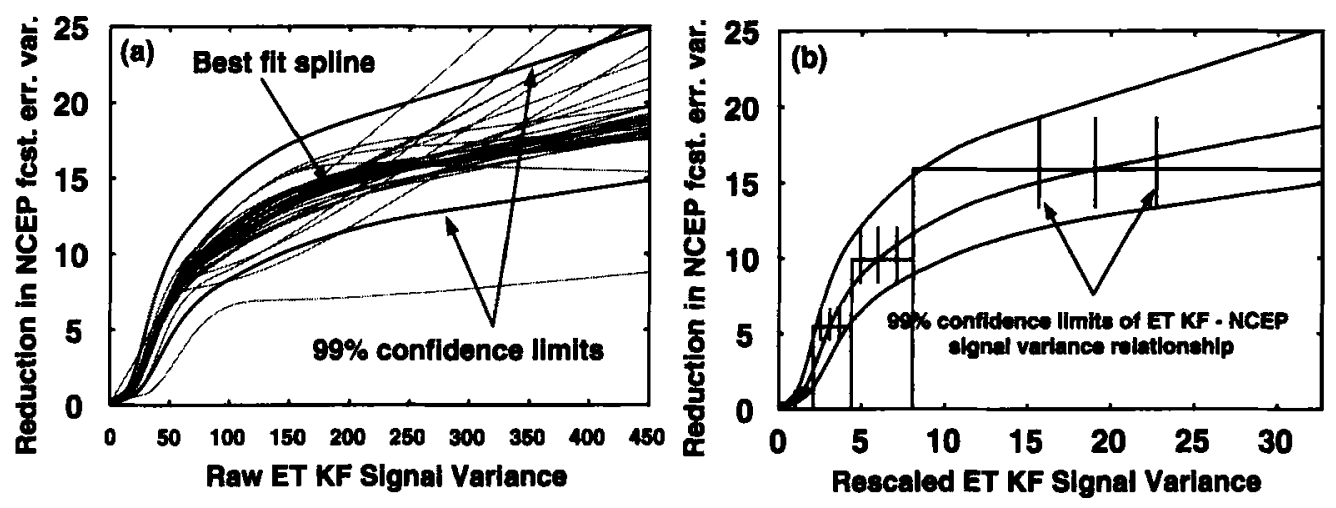

Figure 7. (a) Thin lines: cubic splines for the raw ET KF signal variance reduction in NCEP forecast-error variance relationship in which data from each of the 30 verification regions have been suppressed. The splines are calculated using the relationships shown in Figs. 4(c) and 6. Thick lines: best-fit cubic spline and the splines that pass through the 99\% confidence limits for the full dataset (using Figs. 4(b) and 5(f)). (b) 99\% confidence limits and best-fit cubic spline for the reduction in NCEP forecast-error variance as a function of the ET KF signal variance, rescaled by a factor of roughly 14 .

\section{CONCLUSIONS AND DISCUSSION}

The ability of the ET KF to predict (i) the NCEP signal variance and (ii) the reduction in NCEP forecast-error variance due to targeted dropwindsonde observations was tested, in a first step towards quantifying predictability. A linear, increasing relationship between the ET KF and NCEP signal variances was found to exist (i) over all observation locations selected on 12 distinct observing days during WSRO0, and (ii) over all 30 chosen verification regions of interest, at the verification time. However, the ET KF consistently overpredicted the operational signal variance by a factor of roughly 8 at the analysis (targeting) time and 14 at the verification time. A statistical rescaling factor was therefore introduced to correct the ET KF's prediction of signal variance. Furthermore, a monotonically increasing, but nonlinear, relationship was found to exist between the NCEP forecast-signal variance and the reduction in NCEP forecast-error variance. Using the rescaling factor of 14 , the forecast-error variance reducing effect of targeted observations was graphed as an approximate function of the predicted ET KF signal variance.

The discrepancy between the rescaling factors at the analysis and verification times indicates that the ET KF signal variance structures grew nearly twice as fast as the NCEP signal realizations did between these times, on average. Our reasoning for the discrepancy is that signals produced by an ET KF data-assimilation scheme are able to project significantly onto growing structures in the vicinity of the observation locations, whereas signals produced by a quasi-isotropic data-assimilation scheme such as 3D-Var usually tend to be localized near observation sites, with relatively little projection onto growing structures, and perhaps significant projection onto neutral and decaying structures.

A more detailed analysis of the statistical significance of the relationship between ET KF and NCEP signal variances, and reduction in NCEP forecast-error variance, is beyond the scope of this paper. Using a limited sample from WSR00, we have simply illustrated how the results of targeting experiments can be used retrospectively to obtain functions that map (i) ET KF signal variance predictions onto NCEP signal 
variance, (ii) NCEP signal variance onto reduction in NCEP forecast-error variance, and (iii) ET KF signal variance predictions onto reduction in NCEP forecast-error variance.

Several potential benefits arise from the ET KF's ability to make rapid quantitative predictions of operational signal variance and error variance reduction:

(i) There is increased confidence that the ET KF can discriminate between aircraft deployments that have high and low potential for reducing forecast error. Moreover, the ET KF may additionally be able to predict the relative benefits to forecasts of mobile observational platforms deployed at different times. For example, if a strategy of aircraft deployments over the next 1-3 days can be communicated to flight planning officials in advance, this considerably eases the logistics of air traffic control and fuel and crew management. Via a dynamic approach based on 'serial targeting' (described in Bishop et al. (2001) and MEA), the ET KF could also be used to deploy combinations of adaptive observing platforms that take observations at more regular assimilation intervals. Relevant new platforms such as driftsondes and aerosondes are being proposed in the planning of future targeted observing missions.

(ii) Operational data quality-control schemes automatically reject new observations if they differ significantly from the background field. A large ET KF signal variance at an observation site would suggest that the observation and background field are more likely to differ significantly than if the ET KF signal variance were small. In such cases, the ET KF could therefore be used to prevent data quality-control schemes from throwing out data simply because they differed greatly from the background field. These new observed data could be of immense value to an analysis, especially in frontal regions.

(iii) The cost benefit of forecast improvement from any deployment of observational resources could be predicted, by coupling predictions of forecast-error variance reduction made using the ET KF with a simple economic model that relates forecast-error reduction to units of currency saved in populated regions. In this way, optimal combinations of deployments over different future times could be prioritized, based on the relative human and economic importance of major weather events in selected regions.

The ET KF estimation of operational forecast-error variance reduction can be improved beyond a simple statistical rescaling. Firstly, improvements to the presently crude estimates of ET KF analysis-error covariance are required. Secondly, the problem of rank deficiency of ET KF forecast-error covariance estimates needs to be addressed. Thirdly, the accuracy of error covariances specified in the ET KF depends on the ensemble generation technique. Several such techniques exist, for example, bred (or evolved) perturbations, singular vectors and perturbed observations. Another possibility is to use the ET KF itself to generate perturbations. A quantitative test of the capability of each technique in predicting signal variance is required.

Fourthly and most significantly, the background-error covariances currently assumed by the NCEP 3D-Var data-assimilation scheme and those assumed by the ET KF are very different. More advanced data-assimilation schemes such as 4D-Var (Janisková et al. 1999; Rabier et al. 2000) are expected to produce error covariances that better resemble those produced using ensemble perturbations. Evensen and van Leeuwen (1996), Houtekamer and Mitchell (1998), Mitchell and Houtekamer (2000), Anderson and Anderson (1999), Hamill and Snyder (2000) and Bishop et al. (2001) have all proposed data-assimilation schemes that attempt to improve background-error statistics by using an outer product of ensemble perturbations. It is expected that dissimilarities between error covariances in the ET KF and these new ensemble-based schemes would be less than those between the ET KF and current quasi-isotropic 3D-Var data-assimilation schemes. 
Regardless of the form of future data-assimilation schemes, the method of statistically correcting the ET KF predictions described in this paper provides a means by which past targeted observations can be used to improve the reliability of ET KF predictions of the error-reducing efficacy of future targeted observations.

\section{ACKNOWLEDGEMENTS}

S. J. Majumdar and C. H. Bishop and B. J. Etherton gratefully acknowledge the financial support of National Science Foundation Grants ATM 96-12502 and ATM 9814376. The authors are also grateful to an anonymous referee for comments that helped improve the manuscript.

Anderson, J. L. and Anderson, S. L. 1999

Bishop, C.H. and Toth, Z.

Bishop, C. H., Etherton, B. J. and Majumdar, S. J.

Buizza, R., Petroliagis, T., Palmer, T. N., Barkmeijer, J., Hamrud, M., Hollingsworth, A., Simmons, A. and Wedi, $\mathbf{N}$.

Burgers, G., van Leeuwen, P. J. and Evensen, $\mathrm{G}$.

Efron, $B$.

Evensen, G.

Evensen, G. and van Leeuwen, P. J. 1996

Hamill, T. M. and Snyder, C.

Holland, G. J., McGeer, T. and Youngren, $\mathbf{H}$.

Houtekamer, P. L. and Mitchell, H. L.

Janisková, M., Veersé, F., Thépaut, J.-N., Desroziers, G. and Pouponneau, B.

Katz, R. W. and Murphy, A. H.

Langland, R. H., Toth, Z., Gelaro, R., Szunyogh, I., Shapiro, M. A., Majumdar, S. J., Morss, R. E., Rohaly, G. D., Velden, C., Bond, N. and Bishop, C. H. Majumdar, S. J., Bishop, C. H., Etherton, B. J. and Toth, Z.

Mitchell, H. L. and Houtekamer, P. L.

Molteni, F., Buizza, R., Palmer, T. N. and Petroliagis, $T$.

1999

2001

1998

1998

2000

1998

\section{REFERENCES}

A Monte Carlo Implementation of the nonlinear filtering problem to produce ensemble assimilations and forecasts. Mon. Weather Rev., 127, 2741-2758

Ensemble transformation and adaptive observations. J. Atmos. Sci., 56, 1748-1765

Adaptive sampling with the ensemble transform Kalman filter. Part I: Theoretical aspects. Mon. Weather Rev., 129, 420-436

Impact of model resolution and ensemble size on the performance of an ensemble prediction system. Q.J.R. Meteorol. Soc., $124,1935-1960$

Analysis scheme in the ensemble Kalman filter. Mon. Weather Rev. 126, 1719-1724

The Jackknife, the Bootstrap and other resampling plans. Society for Industrial and Applied Mathematics (SIAM)

Sequential data assimilation with a non-linear quasi-geostrophic model using Monte Carlo methods to forecast error statistics. J. Geophys. Res., 99(C5), 10143-10162

Assimilation of Geosat altimeter data for the Agulhas current using the ensemble Kalman filter with a geostrophic model. Mon. Weather Rev., 124, 85-96

A hybrid ensemble Kalman filter/3D-variational analysis scheme. Mon. Weather Rev., 128, 2905-2919

1992 Autonomous aerosondes for economical atmospheric soundings anywhere on the globe. Bull. Am. Meteorol. Soc., 73, 19871998

Adaptive sampling with the ensemble transform Kalman filter Part II: Field program implementation. Mon. Weather Rev., in press

An adaptive ensemble Kalman filter. Mon. Weather Rev, 128, $416-433$

1996 The ECMWF ensemble prediction system: Methodology and validation. Q. J. R. Meteonol. Soc., 122, 73-120 
Parrish, D. F. and Derber, J. C.

1992

Rabier, F., Jarvinen, H., Klinker, E., 2000 Mahfouf, J.-F. and Simmons, A.

Szunyogh, I., Toth, Z., Emanuel, K. A., Bishop, C. H., Snyder, C., Morss, R. E., Woolen, J. S. and Marchok, T. P.

Szunyogh, I., Toth, Z. Majumdar, S. J., Morss, R. E., Bishop, C. H. and Lord, S. J.

Szunyogh, I., Toth, Z., Morss, R. E., 2000 Majumdar, S. J., Etherton, B. J. and Bishop, C. H.

Toth, Z. and Kalnay, E.

Toth, Z., Szunyogh, I., Majumdar, S. J., Morss, R. E., Etherton, B. J., Bishop, C. H. and Lord, S. J.

Toth, Z., Szunyogh, I., Majumdar, S. J., Morss, R. E. Etherton, B. J., Bishop, C. H., Lord, S. J., Ralph, M., Persson, $\mathrm{O}$. and $\mathrm{Pu}, \mathrm{Z}$

Toth, Z., Szunyogh, I., Bishop, C. H., Majumdar, S. J., Morss, R. E. and Lord, S. J.

Wu, W.-S. and Joo, S.-W.
$1999 \mathrm{a}$

$1999 b$

1997
The National Meteorological Center's spectral statisticalinterpolation analysis system. Mon. Weather Rev., 120, 1747-1763

The ECMWF operational implementation of four-dimensional variational assimilation I: Experimental results with simplified physics. O. J. R. Meteorol. Soc., 126, 1143-1170

Ensemble-based targeting during FASTEX: The impact of dropsonde data from the LEAR jet. $Q$. J. R. Meteorol. Soc., 125, 3189-3217

'Ensemble-based targeted observations during NORPEX'. Pp. 74-77 in Preprints of the 3rd AMS symposium on integrated observing systems, 10-15 January 1999, Dallas TX, USA. American Meteorological Society

The effect of targeted dropsonde observations during the 1999 Winter Storm Reconnaissance Program. Mon. Weather Rev., 128, 3520-3537

Ensemble forecasting at NCEP and the breeding method. Mon. Weather Rev., 125, 3297-3319

'The 1999 Winter Storm Reconnaissance Program'. Pp. 27-32 in Preprints of the 13th AMS conference on numerical weather prediction, 13-17 September 1999, Denver CO, USA. American Meteorological Society

'Targeted observations at NCEP: Toward an operational implementation'. Pp. 186-193 in Preprints of the 4th AMS symposium on integrated observing systems, 10-14 January 2000 Long Beach CA, USA. American Meteorological Society

'On the use of targeted observations for improving numerical weather forecasts'. Pp. 72-79 in Preprints of the 5th AMS symposium on integrated observing systems, 15-19 January 2001, Albuquerque NM, USA. American Meteorological Society

The change of the observational errors in the NCEP SSI analysis ${ }^{*}$ Pp. 84-85 in Preprints of the 11th AMS Conference on Numerical Weather Prediction, 19-23 August 1996, Norfolk VA, USA. American Meteorological Society 